\title{
Analyzing Secondary Metabolite Production by 3D Printed Bacterial Populations Using Scanning Electrochemical Microscopy
}

\author{
Jodi Connell $^{1,3}$, Jiyeon Kim ${ }^{2}$, Jason B. Shear ${ }^{2}$, Allen J. Bard ${ }^{2}$, and Marvin Whiteley, ${ }^{1,3}$ \\ ${ }^{1}$ Department of Molecular Biosciences, University of Texas at Austin, Texas, USA \\ ${ }^{2}$ Department of Chemistry, University of Texas at Austin, Texas, USA \\ ${ }^{3}$ Center for Infectious Disease, University of Texas at Austin, Texas, USA
}

Bacteria communicate via short-range physical and chemical signals, interactions known to mediate quorum sensing, sporulation, and other adaptive phenotypes. Although most in vitro studies examine bacterial properties averaged over large populations, the levels of key molecular determinants of bacterial fitness and pathogenicity (e.g., oxygen, quorum-sensing signals) may vary over micrometer scales within small, dense cellular aggregates believed to play key roles in disease transmission [1]. A detailed understanding of how cell-cell interactions contribute to pathogenicity in natural, complex environments will require a new level of control in constructing more relevant cellular models for assessing bacterial phenotypes.

Here, we describe a microscopic three-dimensional (3D) printing strategy [2] that enables multiple populations of bacteria to be organized within essentially any 3D geometry, including adjacent, nested, and free-floating colonies (Fig. 1a). In this laser-based lithographic technique, microscopic containers are formed around selected bacteria suspended in gelatin via focal cross-linking of polypeptide molecules. After excess reagent is removed, trapped bacteria are localized within sealed cavities formed by the cross-linked gelatin, a highly porous material that supports rapid growth of fully enclosed cellular populations and readily transmits numerous biologically active species, including polypeptides, antibiotics, and quorum-sensing signals. Using this approach, we show that a picoliter-volume aggregate of Staphylococcus aureus can display substantial resistance to $\beta$-lactam antibiotics by enclosure within a shell composed of Pseudomonas aeruginosa.

This novel 3D printing technology provides the unprecedented ability to organize multiple populations at defined positions and distances to investigate key questions regarding the spatial requirements for bacterial interactions and intercellular communication. This highly adaptable method can be interfaced with other emerging analytical techniques to establish new experimental platforms for characterizing how spatial organization impacts interactions within microbial populations at the molecular level. Here, we introduce one example of the many conceivable integrated approaches, where this 3D printing strategy is used in combination with scanning electrochemical microscopy (SECM) to create a quantitative, spatiotemporal map of the production of a quorum-sensing-regulated redox-active secondary metabolite, pyocyanin [3], by $P$. aeruginosa in confined aggregates containing $\leq 10^{4}$ cells in real-time with microscale $3 \mathrm{D}$ resolution.

The initial studies presented here, probing the onset of pyocyanin production as small populations of $P$. aeruginosa grow over time as well as the distances over which neighboring communities of mutant strains can sense and respond to one another (Fig. 1b and 1c), establish the utility of this integrated approach for characterizing how spatial parameters influence microbial behaviors. 


\section{References:}

[1] Connell JL, Wessel AK, Parsek MR, Ellington AD, Whiteley M, Shear JB. MBio 1. (2010)

[2] Connell JL, Ritschdorff ET, Whiteley M, Shear JB. PNAS. 110:18380-18385. (2013)

[3] D. Koley, M.M. Ramsey, A.J. Bard, and M. Whiteley. PNAS. 108(50):19996-20001. (2011)

[4] This work was supported by NIH grant 1RO1AI075068 (to M.W.), and has benefited from image analysis developments supported by NIH grant R03AI081216 (to J.B.S. and M.W.).
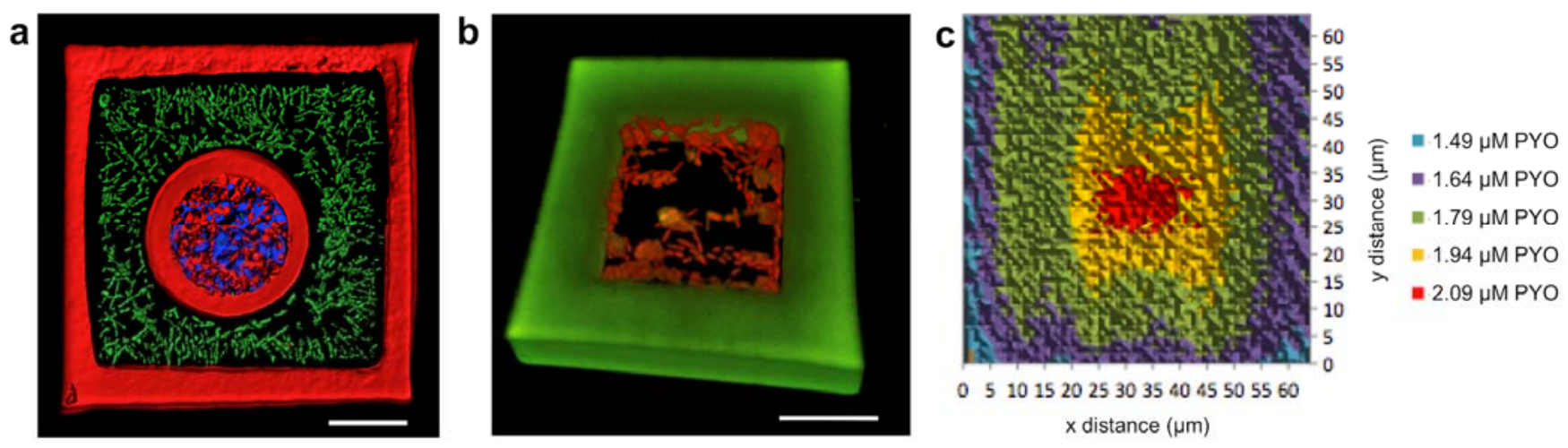

Figure 1. a) Gelatin-based micro-3D printing of a spatially structured polymicrobial community. A confocal fluorescence isosurface with the roof of each microtrap cut away shows a spatially organized polymicrobial community constructed using gelatin-based micro-3D printing, where a nested population of $S$. aureus (blue) is surrounded on all sides (except on the coverglass) by P. aerugniosa cells (green). The photocrosslinked walls printed to confine each population appear red. b-c) Scanning electrochemical microscopy (SECM) of pyocyanin production within confined aggregates of $P$. aeruginosa. b) A confocal fluorescence image shows $P$. aeruginosa cells (red) confined within an 8-pL microtrap. c) Spatial map of the pyocyanin (PYO) concentration measured $3 \mu \mathrm{m}$ above the 8-pL microtrap containing $\sim 800 \mathrm{P}$. aeruginosa cells shows a gradient of PYO directly above the cells, where the highest concentration $(2.09 \mu \mathrm{M}$ PYO) is observed in the center of the trap. Scale bars, $10 \mu \mathrm{m}$. 